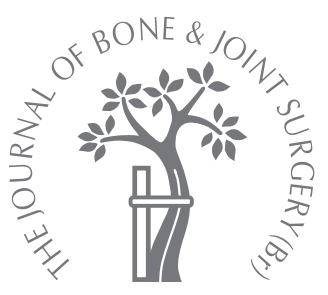

M. R. Chen, J. I. Huang, B. N. Victoroff, D. R. Cooperman

From Case Western Reserve University, Obio, United States
M. R. Chen, MD, Resident

B. N. Victoroff, MD, Associate

Professor

D. R. Cooperman, MD,

Professor

Department of Orthopaedic

Surgery

Case Western Reserve

University, 11100 Euclid

Avenue, Cleveland, Ohio 44118

USA.

II. I. Huang, MD, Assistant Professor

Department of Orthopaedics and Sports Medicine

University of Washington

Medical Center, 4245 Roosevelt

Way NE, Seattle, Washington

98105, USA.

Correspondence should be sent to $\operatorname{Dr}$ M. R. Chen; e-mail: mikechenmd@yahoo.com

(C)2010 British Editorial Society of Bone and Joint Surgery doi:10.1302/0301-620X.92B1.

$22678 \$ 2.00$

$J$ Bone Joint Surg [Br]

2010;92-B:164-8.

Received 2 April 2009;

Accepted 11 August 2009

RESEARCH

\title{
Fracture of the clavicle does not affect arthritis of the ipsilateral acromioclavicular joint compared with the contralateral side
}

\author{
AN OSTEOLOGICAL STUDY
}

\begin{abstract}
In an osteological collection of $\mathbf{3 1 0 0}$ specimens, $\mathbf{7 0}$ were found with unilateral clavicular fractures which were matched with $\mathbf{7 0}$ randomly selected normal specimens. This formed the basis of a study of the incidence of arthritis of the acromioclavicular joint and the effect of clavicular fracture on the development of arthritis in the ipsilateral acromioclavicular joint. This was graded visually on a severity scale of 0 to 3 . The incidence of moderate to severe arthritis of the acromioclavicular joint in normal specimens was $77 \%$ (100 specimens). In those with a clavicular fracture, 66 of 70 (94\%) had arthritis of the acromioclavicular joint, compared to 63 of $70(90 \%)$ on the non-injured contralateral side $(p=0.35)$.

Clavicles with shortening of $15 \mathrm{~mm}$ or less had no difference in the incidence of arthritis compared to those with shortening greater than $15 \mathrm{~mm}(p=0.25)$. The location of the fracture had no effect on the development of arthritis.
\end{abstract}

Primary arthritis of the acromioclavicular (AC) joint is common in the general population, with an incidence as high as $82 \%$ in asymptomatic subjects. ${ }^{1}$ Post-traumatic arthritis is believed to be common following distal clavicular fractures, particularly those with an intra-articular component. ${ }^{2}$ However, the majority of patients with such fractures remain asymptomatic. ${ }^{3}$ Following grade I and II sprains of the AC joint, up to $42 \%$ of patients develop symptoms. ${ }^{4}$

It is not known whether malunited fractures of the clavicle result in an increased incidence of ipsilateral arthritis of the AC joint. In a small series, Edelson ${ }^{5}$ found no evidence of arthritic changes in the adjacent $\mathrm{AC}$ joint in specimens with fractures of the clavicle. Based on these findings, shortening of the clavicle after fracture was suggested to provide a 'physiologic arthroplasty' which protects the joint from arthritic change. ${ }^{5}$ This study evaluates the incidence of arthritis of the AC joint and the effect of clavicular fracture on its development. We hypothesise that such a fracture does not affect the incidence of ipsilateral arthritis of the AC joint compared to the contralateral side.

\section{Materials and Methods}

The clavicles and scapulae of 3100 specimens from the Hamann-Todd Osteological Collection at the Cleveland Museum of Natural History (Cleveland, Ohio) were examined for evidence of fractures of the clavicle. The specimens consist of the remains of the unclaimed dead in the Cuyahoga County Mortuaries and local hospitals between 1893 and 1938.

Demographic information including age at death, gender, race and the cause of death were available in a database at the museum. Fractures of the clavicle were identified in 90 specimens. Bilateral fractures in three, a missing contralateral clavicle in two, nonunion in three, and broken specimens in 12 were excluded, leaving 70 unilateral fractures for analysis. A total of 70 normal specimens, with 140 AC joints, over 30 years of age at the time of death, were selected at random to serve as controls to determine the incidence of arthritis of this joint in the general population.

The fractured clavicles were analysed for the location of the injury and the amount of shortening that had occurred with healing. The lengths of the fractured and unfractured clavicles were measured on an osteometric board equipped with digital calipers (Mitutoyo Co., Kangawa, Japan). Each clavicle was aligned with its sternal facet flat against the vertical measuring surface. The length of each was measured as the distance from the sternal facet to the most lateral aspect of the clavicle. Shortening was defined as the difference in length between the fractured and contralateral, unfractured clavicle. The location of the fracture was recorded according to the OTA classification ${ }^{6}$ as medial end, diaphysis, or lateral end. 


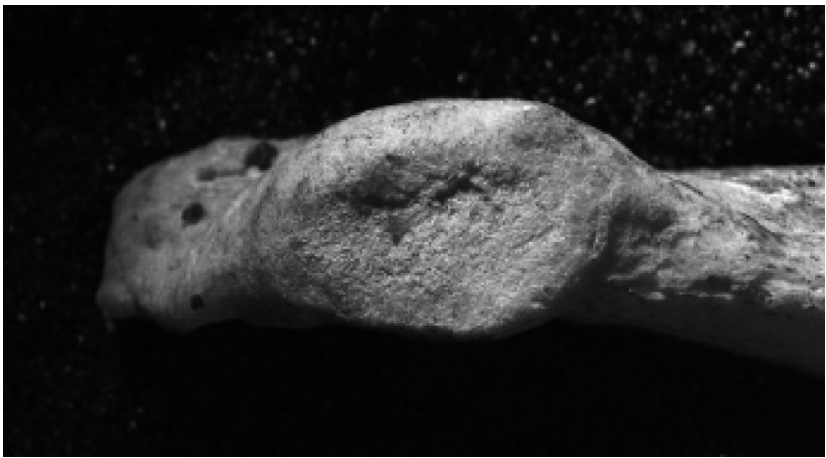

Fig. 1a

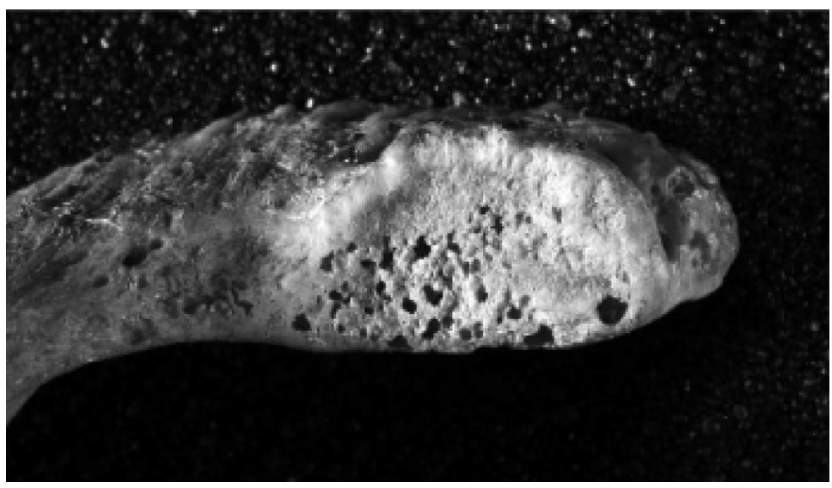

Fig. 1c

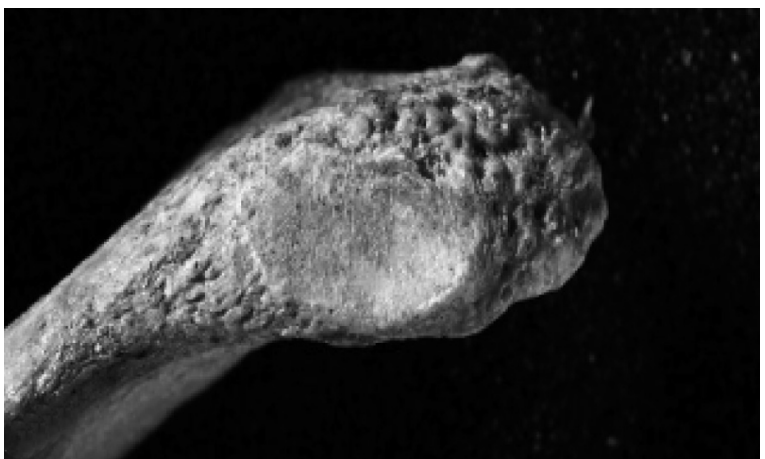

Fig. 1b

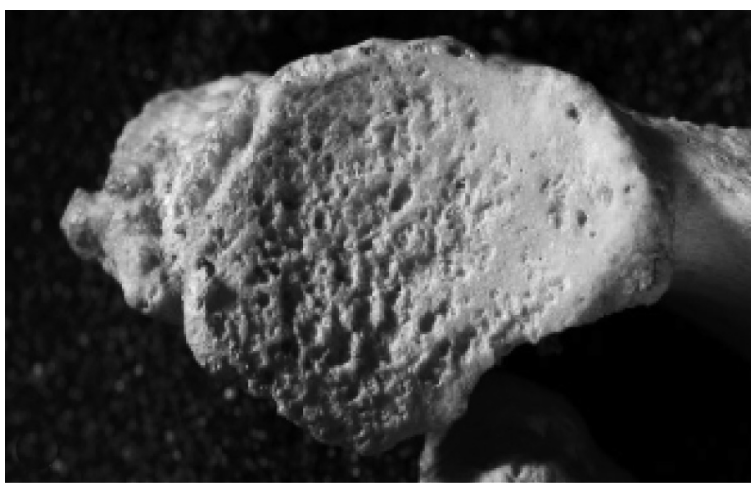

Fig. 1d

Photographs showing grades of acromial arthritis a) grade 0, b) grade 1, c) grade 2, and d) grade 3 .

Arthritis in each clavicle and acromion was assessed visually and by direct palpation. Arthritis was graded on a scale of 0 to 3 (Figs 1 and 2). Grade 0 was defined as no arthritic changes; grade 1 was defined as mild arthritis represented by elongation of the facet or erosion or degeneration of less than $50 \%$ of the facet, but an otherwise smooth joint; grade 2 was defined as moderate arthritis, represented by loss of definition of the facet, the presence of marginal osteophytes, erosion of greater than $50 \%$ of the facet, or joint surface irregularity; grade 3 was defined as severe arthritic changes with large osteophytes present. A single unblinded observer (MRC) performed the grading of all specimens. Arthritic changes in each clavicle and acromion were initially evaluated separately prior to grading arthritic changes at the $\mathrm{AC}$ joint as a combination of the changes in the clavicle and acromion. If the grade of arthritis in the clavicle and acromion differed, that of the AC joint was graded at the higher level.

Statistical analysis. The previously fractured and normal specimens were compared using Student's $t$-test. For statistical analysis, samples were divided into no arthritis (grades 0 and 1) and arthritis present (grades 2 and 3). Statistical analysis for comparison of the presence of arthritis was done with the chi-squared test. For calculations with insufficient expected values, Fisher's exact test was performed. A p-value $<0.05$ was considered statistically significant. In addition, the influence of advancing age on the incidence of arthritis was examined with Pearson's correlation coefficient.
Intra-observer reliability was assessed using Cohen's $\kappa$ analysis, with the observer grading the arthritis in ten different specimens on three separate occasions at one-week intervals. Intra-observer reliability was 0.63 . Inter-observer reliability ${ }^{7}(\kappa)$ was determined by two different observers, in order to demonstrate reproducibility grading arthritis in ten different specimens and finding an inter-observer reliability of 0.6. None of the inter-observer classifications differed by more than one grade.

\section{Results}

Specimens. Those with unilateral fractures were a mean of 58.4 years (33 to 87 ) old at the time of death. There were 62 men and eight women, of whom 57 were Caucasian, 11 were black, one was American Indian, and one was Asian/other. There were 37 clavicles from the left side and 33 from the right. The position of the fracture was in the diaphysis in 60, the medial aspect in three and the lateral aspect in seven. The mean change in length of the fractured clavicle compared to the unfractured clavicle was $11.1 \mathrm{~mm}$, which involved shortening in 66 and lengthening in four. The mean shortening was $12.1 \mathrm{~mm}$ (1.8 to 36.6) and the mean lengthening was $4.08 \mathrm{~mm}$ (2.1 to 9.1).

The 70 randomly selected control specimens without fractures of the clavicle were a mean of 54.0 years (33 to 96) old at death. There was no significant difference in age between the population control specimens and the 


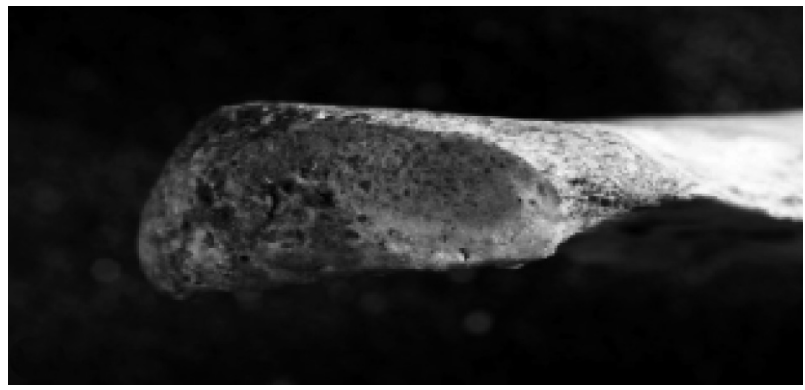

Fig. 2a

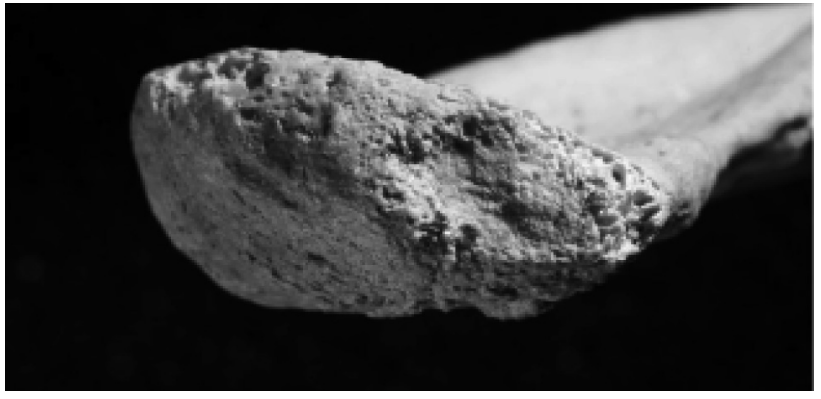

Fig. 2c

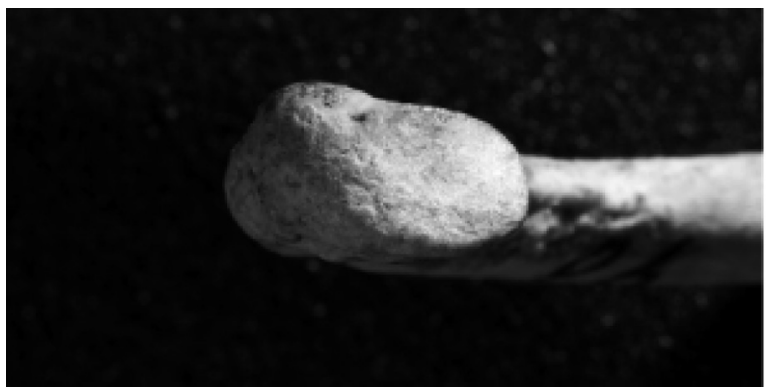

Fig. $2 b$

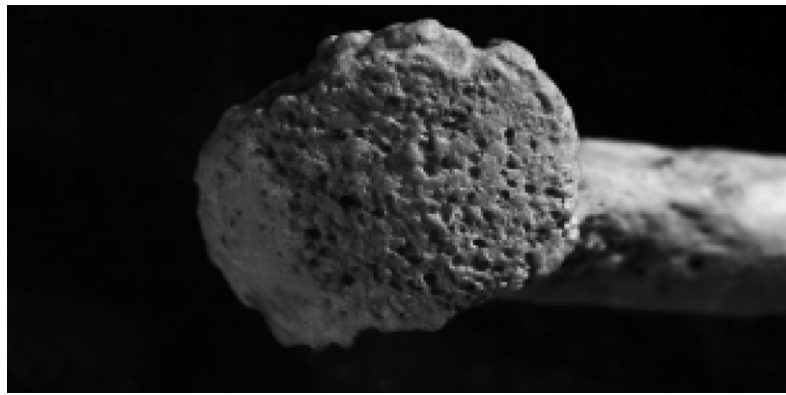

Fig. 2d

Photographs showing grades of clavicular arthritis a) grade 0, b) grade 1, c) grade 2 and d) grade 3.

specimens with a clavicle fracture $(\mathrm{p}=0.09)$. There were 58 male specimens and 12 female, of whom 59 were Caucasian and 11 were black.

AC joint arthritis. The incidence of moderate to severe arthritis of the AC joint in normal specimens was 77\% (100 specimens). Only 14 (7\%) of the AC joints had no evidence of arthritic changes. Moderate to severe arthritis was found in $66(94 \%)$ of the specimens with a previously fractured clavicle, compared to 63 of $70(90 \%)$ in the uninjured contralateral side. There was no significant difference in the presence of arthritis when the fractured and unfractured sides were compared $(\mathrm{p}=0.35)$. There was significantly more moderate to severe arthritis in the AC joints of specimens with a fracture of the clavicle, as well as in the contralateral unfractured side, than in the AC joints of the control specimens without fractures $(\mathrm{p}<0.001)$.

Age. The presence of moderate to severe arthritis of the AC joint in the cohort of normal specimens increased significantly with each decade of age $(p<0.001)$. It was found on $12(40 \%)$ specimens less than 40 years and $13(59 \%)$ aged 40 to 49 years of age. Overall, arthritis occurred in $25(48 \%)$ specimens less than 50 years of age (Fig. 3). The incidence increased to $92 \%(24)$ and $98 \%$ (15), respectively, for specimens 50 to 59 years of age and over 60 years of age. However, there was no significant difference in the incidence of arthritis between the 50 to 59 -year-old group and the $>60$-year-old group $(\mathrm{p}=0.55)$. Even though arthritis was more common in older specimens, older age did not correlate with higher grades of arthritis $(\mathrm{r}=0.11, \mathrm{p}=0.36)$.

In specimens with a clavicular fracture, age did not appear to correlate with the presence of moderate to severe arthritis of the AC joint $(r=0.11, p=0.07)$. Even in specimens younger than 50 years at the time of death there was a higher incidence of arthritis, but this was similar in both fractured and unfractured sides, occurring in 20 of 23 specimens with a clavicular fracture compared to 46 of 47 of the joints on the contralateral side $(\mathrm{p}=0.17)$ (see Fig. 1). Arthritis of the AC joint was found in 20 of $23(87 \%)$ specimens less than 50 years of age, compared to 46 of $47(98 \%)$ specimens over 50 years of age $(\mathrm{p}=$ 0.06). However, compared to the control specimens, the presence of a clavicular fracture did appear to increase the incidence of both ipsilateral and contralateral arthritis in the fracture specimens $(\mathrm{p}<0.01)$.

Location of the fracture. The location of fractures reflected those previously described ${ }^{8}$ with diaphyseal injuries being the most common, followed by lateral and then medial fractures. However, the location of the fracture appeared to have no influence on the presence of moderate to severe arthritis of the AC joint. Of the diaphyseal fractures, 57 of $60(95 \%)$ had arthritis, compared to 54 of $60(90 \%)$ of the contralateral side $(\mathrm{p}=0.30)$. In six of seven lateral fractures arthritis was present with the same incidence on the contralateral side. All three medial fractures had arthritis with the same incidence on the contralateral side. 


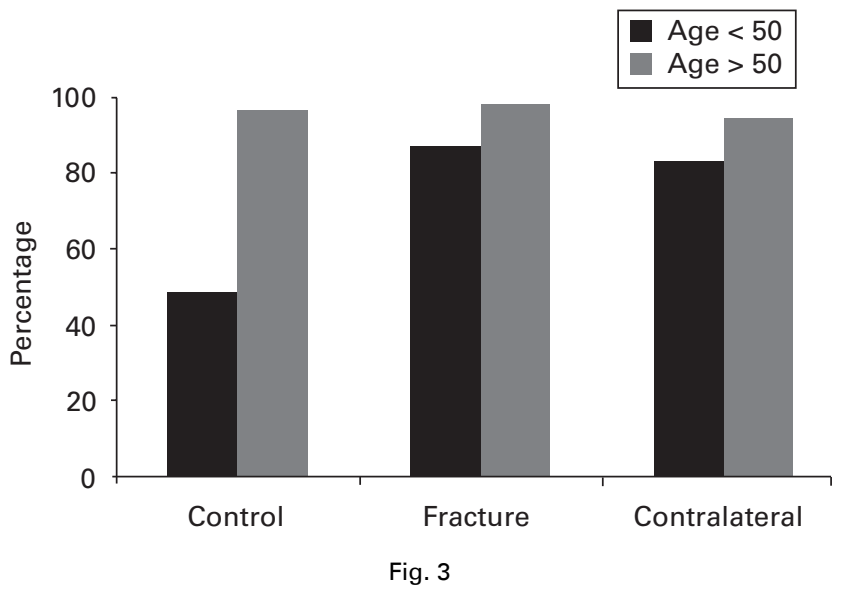

Bar chart showing the incidence of acromioclavicular arthritis.

Fracture length. Shortening due to the fracture did not affect the presence of moderate to severe arthritis. Arthritis was found in 46 of $48(96 \%)$ fractures which had shortened $<15 \mathrm{~mm}$, compared to 16 of $18(89 \%)$ of fractures with shortening of $\geq 15 \mathrm{~mm}$ ( $\mathrm{p}=0.29$ ). The joints in specimens which had shortened $<15 \mathrm{~mm}$ did not differ significantly from the contralateral side (46 of 48 (96\%) vs 43 of $48(90 \%)(\mathrm{p}=0.24)$. Those in specimens which had shortened $\geq 15 \mathrm{~mm}$ had the same extent of arthritis as on the contralateral side (16 of $18,89 \%$ ). Lengthening of the fracture site also did not appear to have an effect on ipsilateral arthritis, as the degree of degenerative change was identical to that on the contralateral side.

\section{Discussion}

The AC joint is a frequent source of shoulder pain, particularly secondary to degenerative arthritis or trauma., Arthritis of the AC joint is prevalent throughout the population. ${ }^{1}$ However, most people are asymptomatic. Recently, there has been interest among trauma and shoulder surgeons in the operative repair of clavicular fractures with a significant deformity because of concern about the resultant functional limitations. ${ }^{9-14}$ The results of this study suggest that malunited clavicular fractures do not lead to an increased risk of degenerative changes in the ipsilateral AC joint, relative to the controlateral unfractured side.

Edelson ${ }^{15}$ has described the bony characteristics of degenerative change in the AC joint. Changes on the acromial side included elongation of the joint and osteophytes, and broadening and rounding-off of the distal clavicle. This study served as the basis for our grading system of AC joint arthritis, which included a visual inspection of the joint surface as well as direct palpation for surface irregularities. As arthritic changes in bone typically follow disc or cartilage degeneration, our grading was probably skewed towards more advanced stages of degenerative change. The joint surface was palpated to detect more subtle irregularities. Osteophytes, which may bridge the joint, were considered more severe as they probably resulted from chronic degeneration. Our grading system demonstrated substantial intra- and inter-observer reliability, similar to that of other visual classifications of arthritis. ${ }^{16}$

A previous study similar to ours, using the Terry Collection at the Smithsonian Museum of Natural History in Washington, DC, noted no evidence of arthritis of the AC joint in nine specimens with clavicular fractures. ${ }^{5}$ In that study, arthritis was defined as bony eburnation, loss of facet definition, and rounding of the distal clavicle. Emphasis was placed on arthrosis across the joint, of which no signs were observed. In fact, the author suggested that clavicular shortening provided a 'physiologic arthroplasty' which helped to dissipate normal loading forces across the acromioclavicular joint. Our larger study demonstrated that the majority of specimens with clavicular fractures had some degree of arthritis. This difference in results may be due to the variation in definitions of arthritis in the joint. Our grading scheme described a progression of degenerative changes exhibited by the AC joint, which differentiated it from the normal joint (Fig. 2).

As expected, arthritis was much more prevalent in the older population, but the severity did not correlate directly with age. In specimens $>50$ years of age the incidence of arthritis was only $48 \%$ in the control specimens, compared to $87 \%$ in the fracture specimen. This suggests that clavicular fractures might have been associated with concomitant injury to the AC joint or contributed to increased load on the joint over time. However, within individual specimens the presence of a clavicular fracture did not appear to affect the incidence of ipsilateral joint arthritis compared to the contralateral side. Interestingly, arthritis was more common on both sides of specimens with clavicular fractures compared to our cohort of normal specimens. We speculate that the fracture alters the biomechanics of the contralateral shoulder girdle to compensate for deformities occurring at the fracture site. Alternatively, there may simply be a demographic bias between the fracture population and the controls. As clavicular fractures usually occur as a result of a high-energy fall, the fracture population may have been more frequently engaged in recreational or occupational activities that predispose them to repetitive load or injuries to the shoulder.

Shortening after fracture was not found to have a significant effect on the development of ipsilateral arthritis. Much debate surrounds the effect of shortening following clavicular fractures, with some studies demonstrating that this results in poor clinical outcomes, ${ }^{11,12,17,18}$ whereas others suggest that shortening has no effect. ${ }^{19-21}$ Unlike high-grade dislocations of the AC joint, which result in significant disruption of the joint, clavicular fractures may not necessarily result in disruption of the acromioclavicular and coracoclavicular ligaments. Thus, the relationship between the distal clavicle and the acromion is maintained. Even if there is resultant widening of the joint following a clavicular fracture, alterations in the mechanics and position of the 
shoulder girdle may also contribute to the maintenance of function in the joint and shoulder. Therefore, the shear forces that contribute to cartilage and bone degeneration in separations of the AC joint may not necessarily exist after fracture of the clavicle.

Traditionally, the site of the fracture, particularly if lateral, was believed to be associated with the development of arthritis of the AC joint. ${ }^{2}$ As in other joints, fractures that affected the joint surface or altered the distribution of forces across adjacent joints often resulted in an increased incidence of arthritis. As the incidence of arthritis of the AC joint was already extremely high in all fracture specimens, and with the small numbers of lateral and medial fractures available, we were not able to test the association of lateral clavicular fractures with the development of ipsilateral AC joint arthritis.

Several limitations are inherent in an anatomical study. First, no clinical information is available about the specimens. Previous studies have demonstrated that the majority of the population has some degree of arthritis of the AC joint, but most people are asymptomatic. ${ }^{22,23}$ It is not known whether the specimens with clavicular fractures had symptomatic AC joints or whether they had functional limitations and the timing of the fracture in relation to the time of death is unknown. Although all fractures were united, it is possible that there may have been a greater effect on arthritis of the $\mathrm{AC}$ joint in specimens which had sustained the fracture earlier in life. Moroever, if clavicular fractures do lead to the future development of ipsilateral arthritis of the AC joint, the chronological progression of this process could not be determined. Arthritis of the AC joint is a common finding, so that specimens with clavicular fractures may have already had pre-existing arthritis and the arthritis observed in the joint arthritis may not have been a result of the clavicular fracture.

No benefits in any form have been received or will be received from a commercial party related directly or indirectly to the subject of this article.

\section{References}

1. Shubin Stein BE, Ahmad CS, Pfaff CH, Bigliani LU, Levine WN. A comparison of magnetic resonance imaging findings of the acromioclavicular joint in symptomatic versus asymptomatic patients. J Shoulder Elbow Surg 2006;15:56-9.
2. Rockwood CA Jr, Williams GR, Young DC. Disorders of the acromioclavicular joint. In: Rockwood CA Jr, Matsen FA III, eds. The shoulder. Vol. 1, Philadelphia: W.B. Saunders, 1990:1:483-553.

3. Nordqvist A, Petersson C, Redlund-Johnell I. The natural course of lateral clavicle fracture: 15 (11-21) year follow up of 110 cases. Acta Orthop Scand 1993;64:87-91.

4. Bergfeld JA, Andrish JT, Clancy WG. Evaluation of the acromioclavicular joint following first- and second-degree sprains. Am J Sports Med 1978;6:153-9.

5. Edelson JG. Clavicular fractures and ipsilateral acromioclavicular arthrosis. J Shoulder Elbow Surg 1996;5:181-5.

6. Orthopaedic Trauma Association Classification, Database and Outcomes committee. Fracture and Dislocation Compendium. J Orthop Trauma 2007;21 (Suppl):572-4.

7. Landis JR, Koch GG. The measurement of observer agreement for categorical data. Biometrics 1977;33;159-74.

8. Robinson CM. Fractures of the clavicle in the adult: epidemiology and classification $J$ Bone Joint Surg [Br] 1998;80-B:476-84

9. No authors listed. Nonoperative treatment compared with plate fixation of dis placed midshaft clavicular fractures: a multicenter, randomized clinical trial. J Bone Joint Surg [Am] 2007;89-A:1-10.

10. Chan KY, Jupiter JB, Leffert RD, Marti R. Clavicle malunion. J Shoulder Elbow Surg 1999;8:287-90.

11. Eskola A, Vainionpaa S, Myllynen P, Pätiälä H, Rokkanen P. Outcome of clavicular fractures in 89 patients. Arch Orthop Trauma Surg 1986;105:337-8.

12. Hill JM, McGuire MH, Crosby LA. Closed treatment of displaced middle-third fractures of the clavicle gives poor results. J Bone Joint Surg [Br] 1997;79-B:537-9.

13. McKee MD, Pedersen EM, Jones $C$, et al. Deficits following nonoperative treatment of displaced midshaft clavicle fractures. J Bone Joint Surg [Am] 2006;88A:35-40.

14. Nowak J, Holgersson M, Larsson S. Sequelae from clavicular fractures are common: a prospective study of 222 patients. Acta Orthop Scand 2005;76:496-502.

15. Edelson JG. Patterns of degenerative change in the acromioclavicular joint. J Bone Joint Surg [Br] 1996;78-B:242-3.

16. Brismar BH, Wredmark T, Movin T, et al. Observer reliability in the arthroscopic classification of osteoarthritis of the knee. J Bone Joint Surg [Br] 2002;84-B:42-7.

17. Lazarides S, Zafiropoulos G. Conservative treatment of fractures of the middle third of the clavicle: the relevance of shortening and clinical outcome. J Shoulder Elbow Surg 2006;15:191-4.

18. Wick M, Muller EJ, Kollig W, Muhr G. Midshaft fractures of the clavicle with a shortening of more than $2 \mathrm{~cm}$ predisposes to nonunion. Arch Orthop Trauma Surg 2001;121:207-11

19. Nordqvist A, Redlund-Johnell I, von Scheele A, Petersson CJ. Shortening of clavicle after fracture: incidence and clinical significance: a 5-year follow-up of 85 patients. Acta Orthop Scand 1997;68:349-51.

20. Nowak J, Holgersson M, Larsson S. Can we predict long-term sequelae after fractures of the clavicle based on initial findings?: a prospective study with nine to ten years of follow-up. J Shoulder Elbow Surg 2004;13:479-86.

21. Oroko PK, Buchan M, Winkler A, Kelly IG. Does shortening matter after clavicle fracture? Bull Hosp Jt Dis 1999;58:6-8.

22. DePalma AF. The role of the disks of the sternoclavicular and acromioclavicular joints. Clin Orthop 1959;13:222-33.

23. DePalma AF, Caller G, Bennett GA. Variational anatomy and degenerative lesions of the shoulder joints. Instr Course Lect 1949;6:255-81. 